\title{
Neuroscience, mental health and the immune system: overcoming the brain-mind-body trichotomy
}

\author{
C. M. Pariante \\ Department of Psychological Medicine, Institute of Psychiatry, Psychology \& Neuroscience, King's College London, London, UK
}

Psychiatry is having a great time. Over the last few years, we have seen an exceptional explosion in neuroscience knowledge, and especially in our understanding of the molecular mechanisms through which environmental and genetic factors affect the brain and regulate behaviour, while at the same interacting with peripheral ('body') functions. While this explosion, and its translational implications, can be seen across a variety of fields, this editorial will focus on one particular area where these developments have been more noticeable: the interaction between neuroscience, mental health and the immune system. This editorial will focus on the broader impact of this discipline as an example of successful translational neuroscience overcoming the brain-mind-body trichotomy.

First published online 27 October 2015

Key words: Brain, depression, immune system, inflammation, mind, stress.

Men ought to know that from the brain and from the brain only arise our pleasures, joys, laughter and jests as well as our sorrows, pains, grieves and tears - Hippocrates

There are more synapses in the human brain than there are stars in our Milky Way galaxy - TED Ed

Psychiatry is having a great time. Over the last few years, we have seen an exceptional explosion in neuroscience knowledge, and especially in our understanding of the molecular mechanisms through which environmental and genetic factors affect the brain and regulate behaviour (Insel \& Landis, 2013), while at the same time interacting with peripheral ('body') functions (Balon, 2015). While this explosion, and its translational implications, can be seen across a variety of fields, this editorial will focus on one particular area where these developments have been more noticeable (and also an area that is particularly dear to the author): the interaction between neuroscience, mental health and the immune system. This area, called psychoneuroimmunology more than 30 years ago (Ader, 1981) and recently renamed immunopsychiatry (Bullmore \& Lynall, 2014), has now started to deliver translational findings relevant to mental health; to etiological mechanisms, diagnosis and treatment. This editorial will also focus on the broader impact of this new discipline as an example of successful translational neuroscience overcoming the brain-mind-body trichotomy.

Address for correspondence: Professor C. M. Pariante, MD, PhD, FRCPsych, Department of Psychological Medicine, Institute of Psychiatry, Psychology \& Neuroscience, King's College London, UK.

(Email: carmine.pariante@kcl.ac.uk)
At the least three consecutive generations of scientists have tried to understand the processes by which immune system participates to the pathogenesis of mental disorders and influence the response to psychotropic medications. However, the recent surge of interest in this research field is due to both an increased clinical knowledge of the role of immune processes in health and diseases, and to a detailed understanding of the molecular and cellular basis of immune-brain communication. Moreover, what I find really interesting about this research field, is that it provides a conceptual framework that integrates genetic, developmental and social factors while being essentially biologically-based; hence it is an example of a successful interaction between neuroscience and mental health.

\section{Of immunity, genes and society}

The prevailing model explaining how the immune system induces psychiatric symptoms builds on the brain, the body and the mind, while also offering convergent mechanisms able to overcome this trichotomy. In a purposely over-simplified summary, increased activation of the peripheral (body) immune system leads to: (1) the production of neurotoxic metabolites of the tryptophan cascade, which is activated both in the brain and in the liver; (2) increased activation of microglia cells (the immune cells of the brain) by circulating cytokines; and (3) changes in brain function via afferents pathways localised in the vagal nerve stimulated peripherally (Dantzer et al. 2008). At a molecular/ cellular level, these alterations result in changes in neurotransmitter functions (increase glutamatergic 
and decreased serotonergic and dopaminergic activity) and an inhibition of neuroplasticity and neurogenesis, that is, of the birth of new neurones and the formation of new dendrites and synapses (Borsini et al. 2015). Undoubtedly, the peripheral ('body') localisation of the primum movens for neuropsychiatric symptoms within this framework is already a quite revolutionary development, overcoming the brain-mind-body tricothomy. However, I believe that the true strength of this model is its ability to integrate genetic and social factors into a single biological framework.

Some gene variants that are associated with increased immune activation are also associated with an increased risk of psychopathology (Bufalino et al. 2013). Indeed, we have shown that immune genes may have larger effects on behavioural outcomes than 'psychiatric genes'. We compared the effects of a polymorphism in the interleukin-6 (IL-6) gene and of the well-known short/long polymorphism in the serotonin transporter on predicting depression during an inflammatory status induced by treatment with interferon-alpha for viral hepatitis (Bull et al. 2009). While the serotonin transporter polymorphism predicts depressive symptoms with an effects size of $0.2-0.3$, the IL-6 polymorphism has an effect size of $0.6-0.8$. The simple notion that an immune gene, which has nothing to do with neurotransmitters, can regulate behaviour, is yet another piece of evidence further overcoming the brainmind-body tricothomy.

Other patients develop increased immune activation (and hence an increased risk of psychopathology) not for a genetic predisposition, but because of environmental factors, including exposure to trauma, social deprivation, or unhealthy nutrition. Exposure to trauma early in life is one of the most established environmental factors increasing the risk for mental disorders, and we have shown that early life trauma activates the immune system in young adults even in the absence of psychopathology (Danese et al. 2007). Interestingly, these young adults with increased immune activation may be at higher risk of developing psychopathology in the future, as shown by prospective studies (see below). Moreover, increased immune activation has been shown following a variety of social adversities, including exposure to personal maltreatment or violence, socioeconomic disadvantage or isolation and other forms of chronic stress (Miller et al. 2008, 2009; Danese et al. 2009; Baumeister et al. 2015a). Of course, we know that exposure to social adversities is a risk factor for the development of mental health problems, and it is tempting to speculate that these two phenomena are causally related: that increased inflammation induced by social adversities increases the risk of psychopathology by altering brain function. We will debate more causation $v$. association below.

Until now we have looked at factors that putatively increase the risk of mental disorders by increasing immune activation; but there are also factors that putatively decrease this risk by reducing immune activation, and nutrition is one of these. Micronutrients associated with high levels of fish intake (polyunsaturated fatty acids, PUFAs) are known to be both preventative and therapeutic in mild/moderate forms of depression and perhaps protective toward the development of psychosis (Su et al. 2015). Both direct action on the brain (increasing neuroplasticity) and indirect action via the immune system (antiinflammatory effects) have been demonstrated for PUFAs, and the latter mechanisms again exemplifies a pathway through which an environmental factor can regulate the mind via the brain and the body: another piece of evidence overcoming the brainmind-body tricothomy.

So, why is the immune system regulated by so many environmental factors, and especially by exposure to stressors early in life? One explanation is that the immune system is 'programmed' to remain hyperactive during the whole life as this confers protection from infections in a high-stress environment (let us not forget that most stressors, until a few hundred years ago, entailed exposure to predators, physical trauma, wounding and infections). Indeed, one may even argue that depression is the 'price to pay' so that our organism survives under conditions of stress (Raison et al. 2006). Obviously today most stressors are psychosocial/relational, and do not entail physical trauma, so the benefits of this mechanism do no longer outweigh the costs; but it is no use arguing with evolution, unfortunately.

\section{Of chicken, egg and causality}

All of the studies mentioned until now are association studies, and do not imply causation; indeed, all this evidence can as easily be interpreted more conservatively as indicating that genetic or social factors are independently associated with both immune activation and (through unknown mechanisms) increased risk of mental disorders. So, how can we try to test the hypothesis that indeed there is a causal relationship between genetics/social factors and mental disorders through immune-related mechanisms?

What if the cause temporarily precedes the effects? Indeed, many longitudinal studies have shown that high levels of inflammation in otherwise healthy individuals predict the future development of psychopathology over the subsequent months or years (Valkanova 
et al. 2013). What if we can induce the effects (psychopathology) by experimentally activating the cause, that is, by increasing immune activation in otherwise healthy individuals? Indeed, administration of an immune activator for experimental or therapeutic purposes can induce neuropsychiatric symptoms, ranging (in a dose-dependent relationship with the severity of inflammation) from transient sadness to clinically significant depression, anxiety, or even (rarely) psychotic symptoms (Capuron \& Miller, 2004; Harrison et al. 2009). What if drugs that are used routinely for the treatment of the effects (psychopathology) have an effect on the cause (the immune system)? Indeed, both antidepressants and antipsychotics have been found to be (on balance, although not unequivocally) immunosuppressant and antiinflammatory (Baumeister et al. 2015b). And, finally, what if we can eliminate the effects (psychopathology) by eliminating the cause (with anti-inflammatory medications)?

Recent systematic reviews and meta-analysis of randomised controlled trials using anti-inflammatory medications in mental disorders (mostly small studies) have shown that adding an anti-inflammatory to an antidepressant or an antipsychotic increases the efficacy of these medications (Fond et al. 2014; Na et al. 2014). But one large study seems to contradict everything (Raison et al. 2013). This trial used a drug for rheumatoid arthritis, Infliximab, as adjuvant to antidepressants in treatment-resistant depressed patients. Overall the study is negative: there is no potentiation of antidepressant effects. However, the study additionally suggests (in a secondary analysis and with results not reaching statistical significance) that there is indeed a potentiation of antidepressant effects in those patients who have higher levels of inflammation at baseline (which, in a way, makes sense). Of note is also the evidence from our studies offering a 'mirrorimage' of these findings, that is, showing that depressed patients with high inflammation do not respond to conventional antidepressants or antipsychotics (Cattaneo et al. 2013; Mondelli et al. 2015). One can see the possible 'personalised treatment algorithm' developing in the future, if and when all of these studies will be replicated: patients with normal levels of inflammation could be reassured that they will respond to first-line antidepressants and antipsychotics, and will be started on routine medications; and patients with increased inflammation could be supported more intensively with personalised treatment algorithms and more assertive, adjuvant strategies, including anti-inflammatories. However, larger trials are needed to test this therapeutic model, and at this stage we ought to discourage patients from taking over the counter anti-inflammatories, especially considering the risk of adverse interactions with psychotropic medications.

\section{Binging together brain, mind and body without losing the primacy of the brain}

Why is psychoneuroimmunology (immunopsychiatry) important, and why have I used it as an example of a successful interaction between neuroscience and mental health? Because it has brought neuroscience and mental health close to medicine again, while at the same time offering a biological framework that can incorporate genetics, social and environmental factors. Even if this research field will not offer the revolutionary treatments of the future, its cultural impact cannot be emphasised enough. By bringing neuroscience and mental health close to medicine again, it has also shown that, while the brain is influenced by the body, the mind is the product of the brain.

Saying that the brain is in the body, and influenced by the body, is an obvious concept. But why saying that the mind is the product of the brain is considered controversial? If we cut the blood supply to the brain, our mind dies: this is perhaps the strongest proof. But if we are hypoglycaemic we faint or convulse, if we have low thyroid hormones or high calcium levels we become depressed, and if we have liver failure or Cushing's syndrome we develop delusions or hallucinations. And, consistently with this notion, high levels of peripheral immune activation makes us depressed, anxious and (possibly) psychotic. All of these effects require changes in the brain.

Hippocrates said this first. There is nothing else beside the brain that generates our emotions, thoughts and behaviour. Unless we believe that mental processers are the product of a spiritual entity-a soul-then all of these functions are in the brain. Is this 'reductionism'? Nobody accuses cardiologists to be reductionist when they defend the primacy on the heart for the circulation of the blood. You stop the heart and there is no circulation. And I do not see vascular surgeons up in arms, accusing cardiologists to be reductionist. Of course the brain is a complex organ, and we do not really understand it yet. Our model for understanding the biology of mental disorder is still at the beginning, and we can only try to understand how this complexity is influenced by a myriad of factors such as genetic make-up, in utero environment, social experience, upbringing, nutrition, poverty, social support, urbanicity-but all of these factors need the brain to exert their effects on the mind: to protect from, or increase the risk for, mental disorders, which are disorders of emotions, thoughts and behaviour. The fact that there are more synapses in the human brain than there are stars in the Milky Way- 
literally-makes super difficult to understand how the brain generates the mind, but this should not-cannotpush us into a defensive position assuming that 'there must be something else besides the brain'. This is what our ancestors thought about lightening, volcanoes, earthquake and eclipses, before science demonstrated that these are not due to the rage of the gods or the breathing of a dragon.

\section{Acknowledgements}

Professor Pariante's research on depression and inflammation is supported by: the grants 'Persistent Fatigue Induced by Interferon-alpha: A New Immunological Model for Chronic Fatigue Syndrome' (MR/ J002739/1) and 'Immuno-psychiatry: a consortium to test the opportunity for immunotherapeutics in psychiatry' (MR/L014815/1; together with GSK), from the Medical Research Council (UK); the National Institute for Health Research (NIHR) Mental Health Biomedical Research Centre in Mental Health at South London and Maudsley NHS Foundation Trust and King's College London; by Johnson \& Johnson as part of a programme of research on depression and inflammation; and by a Wellcome Trust-led consortium that also include Johnson \& Johnson, GSK and Lundbeck.

\section{Conflict of Interest}

None.

\section{Financial Support}

This research received no specific grant from any funding agency, commercial or not-for-profit sectors.

\section{References}

Ader R (1981). Psychoneuroimmunology. Academic Press: New York.

Balon R, Wise TN (ed.) (2015). Clinical Challenges in the Biopsychosocial Interface. Update on Psychosomatics for the 21st Century. Karger: Basel.

Baumeister D, Akhtar R, Ciufolini S, Pariante CM, Mondelli V (2015a). Childhood trauma and adulthood inflammation: a meta-analysis of peripheral C-reactive protein, interleukin-6 and tumour necrosis factor-alpha. Molecular Psychiatry. Jun 2. doi: 10.1038/mp.2015.67. [Epub ahead of print]

Baumeister D, Ciufolini S, Mondelli V (2015b). Effects of psychotropic drugs on inflammation: consequence or mediator of therapeutic effects in psychiatric treatment? Psychopharmacology. Aug 14. doi: 10.1007/s00213-015-4044-5. [Epub ahead of print]
Borsini A, Zunszain PA, Thuret S, Pariante CM (2015). The role of inflammatory cytokines as key modulators of neurogenesis. Trends in Neurosciences 38, 145-157.

Bufalino C, Hepgul N, Aguglia E, Pariante CM (2013). The role of immune genes in the association between depression and inflammation: a review of recent clinical studies. Brain, Behavior, and Immunity 31, 31-47.

Bull SJ, Huezo-Diaz P, Binder EB, Cubells JF, Ranjith G, Maddock C, Miyazaki C, Alexander N, Hotopf M, Cleare AJ, Norris S, Cassidy E, Aitchison KJ, Miller AH, Pariante CM (2009). Functional polymorphisms in the interleukin-6 and serotonin transporter genes, and depression and fatigue induced by interferon-alpha and ribavirin treatment. Molecular Psychiatry 14, 1095-1104.

Bullmore ET, Lynall ME (2014). Immunologic therapeutics and psychotic disorders. Biological Psychiatry 75, 260-261.

Capuron L, Miller AH (2004). Cytokines and psychopathology: lessons from interferon-alpha. Biological Psychiatry 56, 819-824.

Cattaneo A, Gennarelli M, Uher R, Breen G, Farmer A, Aitchison KJ, Craig IW, Anacker C, Zunsztain PA, McGuffin P, Pariante CM (2013). Candidate genes expression profile associated with antidepressants response in the GENDEP study: differentiating between baseline 'predictors' and longitudinal 'targets'. Neuropsychopharmacology 38, 377-385.

Danese A, Pariante CM, Caspi A, Taylor A, Poulton R (2007). Childhood maltreatment predicts adult inflammation in a life-course study. Proceedings of the National Academy of Sciences of the United States of America 104, 1319-1324.

Danese A, Moffitt TE, Harrington H, Milne BJ, Polanczyk G, Pariante CM, Poulton R, Caspi A (2009). Adverse childhood experiences and adult risk factors for age-related disease: depression, inflammation, and clustering of metabolic risk markers. Archives of Pediatrics and Adolescent Medicine 163, 1135-1143.

Dantzer R, O'Connor JC, Freund GG, Johnson RW, Kelley KW (2008). From inflammation to sickness and depression: when the immune system subjugates the brain. Nature Reviews Neuroscience 9, 46-56.

Fond G, Hamdani N, Kapczinski F, Boukouaci W, Drancourt N, Dargel A, Oliveira J, Le Guen E, Marlinge E, Tamouza R, Leboyer M (2014). Effectiveness and tolerance of anti-inflammatory drugs' add-on therapy in major mental disorders: a systematic qualitative review. Acta Psychiatrica Scandinavica 129, 163-179.

Harrison NA, Brydon L, Walker C, Gray MA, Steptoe A, Dolan RJ, Critchley HD (2009). Neural origins of human sickness in interoceptive responses to inflammation. Biological Psychiatry 66, 415-422.

Insel TR, Landis SC (2013). Twenty-five years of progress: the view from NIMH and NINDS. Neuron 80, 561-567.

Miller GE, Chen E, Sze J, Marin T, Arevalo JM, Doll R, Ma R, Cole SW (2008). A functional genomic fingerprint of chronic stress in humans: blunted glucocorticoid and increased NF-kappaB signaling. Biological Psychiatry 64, 266-272.

Miller GE, Chen E, Fok AK, Walker H, Lim A, Nicholls EF, Cole S, Kobor MS (2009). Low early-life social class leaves 
a biological residue manifested by decreased glucocorticoid and increased proinflammatory signaling. Proceedings of the National Academy of Sciences of the United States of America 106, 14716-14721.

Mondelli V, Ciufolini S, Belvederi Murri M, Bonaccorso S, Di Forti M, Giordano A, Marques TR, Zunszain PA, Morgan C, Murray RM, Pariante CM, Dazzan P (2015). Cortisol and inflammatory biomarkers predict poor treatment response in first episode psychosis. Schizophrenia Bulletin 41, 1162-1170.

Na KS, Lee KJ, Lee JS, Cho YS, Jung HY (2014). Efficacy of adjunctive celecoxib treatment for patients with major depressive disorder: a meta-analysis. Progress in Neuro-psychopharmacology and Biological Psychiatry 48, $79-85$.
Raison CL, Capuron L, Miller AH (2006). Cytokines sing the blues: inflammation and the pathogenesis of depression. Trends in Immunology 27, 24-31.

Raison CL, Rutherford RE, Woolwine BJ, Shuo C, Schettler P, Drake DF, Haroon E, Miller AH (2013). A randomized controlled trial of the tumor necrosis factor antagonist infliximab for treatment-resistant depression: the role of baseline inflammatory biomarkers. JAMA Psychiatry 70, 31-41.

Su KP, Matsuoka Y, Pae CU (2015). Omega-3

polyunsaturated fatty acids in prevention of mood and anxiety disorders. Clinical Psychopharmacology and Neuroscience 13, 129-137.

Valkanova V, Ebmeier KP, Allan CL (2013). CRP, IL-6 and depression: a systematic review and meta-analysis of longitudinal studies. Journal of Affective Disorders 150, 736-744. 